\title{
Extended phase space for a spinning particle
}

\author{
S. Zakrzewski \\ Department of Mathematical Methods in Physics, University of Warsaw \\ Hoża 74, 00-682 Warsaw, Poland
}

\begin{abstract}
Extended phase space of an elementary (relativistic) system is introduced in the spirit of the Souriau's definition of the 'space of motions' for such system. Our 'modification' consists in taking into account not only the symmetry (Poincaré) group but also its action on the (Minkowski) space-time, i.e. the full covariant system. This yields a general procedure to construct spaces in which the equations of motion can be formulated: phase trajectories of the system are identified as characteristics on some constraint submanifold ('mass and spin shell') in the extended phase space. Our formulation is generally applicable to any homogeneous space-time (e.g. de Sitter) and also to Poisson actions. Calculations concerning the Minkowski case for non-zero spin particles show an intriguing alternative: we should either accept two-dimensional trajectories or (Poisson) noncommuting space-time coordinates.
\end{abstract}

\section{Introduction}

According to Souriau [1], the space of 'motions' ('histories', 'phase trajectories') of a classical mechanical system has a structure of a symplectic manifold. If the system is isolated, then the space-time symmetry group acts (symplectically) on this manifold. Elementary systems are those for which this action is transitive (such systems 'do not have other structure than their space-time situation' [1]). By the momentum mapping theory, transitive actions correspond to coadjoint orbits of the underlying group (modulo possible cohomological problems, not present in our basic case: Poincaré group).

This is probably the most basic physical application of groups. The symmetry group under question (Poincaré, Galileo, de Sitter,...) determines (by an algorithm) possible types of elementary particles (mass, spin,...) and the set of their motions. However, the motions are described only as abstract points of coadjoint orbits. The algorithm does not provide any description of motions as solutions of 'equations of motion', which we'd expect to be formulated in an appropriate bundle over space-time.

As a way to determine the full model of particle, we introduce in this paper extended phase spaces (in which the equations of motion can be formulated). Like the 'space of motions', an extended phase space is defined as a symplectic 'transitive' space, the transitivity this time being understood with respect to the pair group + space-time rather than to the group alone (we 'represent' not only the infinitesimal generators of the group but also functions on the space-time, in a covariant way). 
The paper is organized as follows. The definition of extended phase spaces is given in Section 2, after a short investigation of the simplest well known case in Section 1. All extended phase spaces are classified in Section 3 (they turn out to be in one-to-one correspondence with the coadjoint orbits of the Lorentz group). The reduction of an extended phase space by fixing value of spin and mass (which relates the extended phase space to a coadjoint orbit of the Poincaré group) is described in Section 4. It turns out that the history of the particle is represented by a 'world tube' rather than world line.

It is convenient to have in mind here also the quantum case. Quantum elementary particles are related to irreducible unitary representations of the space-time symmetry group and the corresponding full description (wave equation) should be (in our approach) related to covariant representations of the pair group + space-time (in this context, such a pair is called a dynamical system; we prefer to avoid this terminology). In Section 5 we explain how the quantum formulation results from the classical one.

In our opinion, it is convenient to use similar language both in the quantum and the classical case. We shall speak about representations, irreducibility, etc. in the classical case (instead of symplectic realizations, transitivity, etc.).

In Section 6 we perform a partial reduction of an extended phase space by fixing the value of spin. The original fibration over space-time does not descend to the quotient, but there exist another one which does it. With respect to the new fibration, the space-time coordinates do not commute, the Poisson bracket being proportional to the spin tensor and inverse proportional to the square of mass.

\section{Homogeneous formulation of mechanics and rela- tivistic spin zero particle}

Let $Q$ denote the configuration space of a non-relativistic mechanical system. A (nonhomogeneous) hamiltonian formulation of dynamics of such a system is given by specifying a (time-dependent) Hamiltonian function $H: \mathbb{R} \times T^{*} Q \rightarrow \mathbb{R}$, which generates the equations of motion:

$$
\dot{x}^{k}=\frac{\partial H}{\partial p_{k}}, \quad \dot{p}_{k}=-\frac{\partial H}{\partial x^{k}} .
$$

Here $T^{*} Q$ denotes the cotangent bundle of $Q$ (the phase space), $\left(x^{k}\right)_{k=1, \ldots, N}$ - some coordinates in $Q,\left(x^{k}, p_{j}\right)$ - the induced coordinates on the phase space and the dot denotes the differentiating with respect to time.

Now consider the extended configuration space $\mathcal{M}:=\mathbb{R} \times Q$ and the hypersurface $C_{H}$ in $T^{*} \mathcal{M}$ given by

$$
C_{H}=\left\{\left(t, x^{1}, \ldots, x^{N}, e, p_{1}, \ldots, p_{N}\right) \in T^{*} \mathcal{M}: e=H(t, x, p)\right\}
$$

('energy' $=$ 'Hamiltonian'), where $t=x^{0}$ is the time variable and $-e=p_{0}$ is the conjugate variable (minus 'energy'). Of course, specifying $H$ is the same as specifying $C_{H}$. It is easy to check that solutions of (11) are in one-to-one correspondence with characteristics on $C_{H}$ (characteristics $=$ the integral curves of the degeneracy distribution of the symplectic form restricted to $C_{H}$ ). The description in terms of $C_{H}$ is said to be the homogeneous 
formulation of hamiltonian dynamics (cf. e.g. [2]). The cotangent bundle $T^{*} \mathcal{M}$ is said to be the extended phase space.

The homogeneous description is particularly useful in the case of a relativistic point particle (with spin zero). In this case $\mathcal{M}$ is just the Minkowski space-time $M$ :

$$
\mathcal{M}:=M \text {. }
$$

For a free particle with mass $m$, the corresponding submanifold of $T^{*} M$ is just the "mass shell':

$$
C_{m}=\left\{\left(x^{0}, x^{1}, x^{2}, x^{3}, p_{0}, p_{1}, p_{2}, p_{3}\right): p^{2}=m^{2}, p_{0}>0\right\} .
$$

Here $p^{2}=g^{k l} p_{k} p_{l}$ is the Lorentz square of the 4 -momentum $\left(g^{k l}\right.$ is the contravariant Lorentz metric).

The Poincaré group - the (connected) group of affine transformations of $M$ leaving $g$ invariant — will be denoted by $G$, its Lie algebra - by $\mathfrak{g}$. The canonical moment map $J: T^{*} M \rightarrow \mathfrak{g}^{*}$ for the action of $G$ on $T^{*} M$ (the lift of the natural action of $G$ on $M$ ) identifies the set of characteristics on $C_{m}$ with the coadjoint orbit in $\mathfrak{g}^{*}$ corresponding to the mass $m$ and spin zero. This gives a natural realization of the abstract points of this coadjoint orbit as trajectories. The equations of motion are encoded in the mass shell $C_{m}$ (which is nothing else but the inverse image of the coadjoint orbit by $J$ ).

We regard the above description as a full model of a (free) relativistic particle with mass $m$ and spin zero. Now we extract its main features in order to pass to a general case. We observe the following three essential properties of the above model (we set $P:=T^{*} M$ ):

1. $P$ is a Hamiltonian $G$-space, in other words,

$$
\text { a complete Poisson map } J: P \rightarrow \mathfrak{g}^{*} \text { is given }
$$

2. $P$ is fibered over $M$ (with coisotropic fibers), i.e.

$$
\text { a complete Poisson map } \pi: P \rightarrow M \text { is given }
$$

3. the following covariance holds: $X_{P}\left(\pi^{*} f\right)=\pi^{*}\left(X_{M} f\right)$, or, equivalently,

$$
\left\{J^{*} X, \pi^{*} f\right\}=\pi^{*}\left(X_{M} f\right)
$$

for $X \in \mathfrak{g}, f \in C^{\infty}(M)$. Here $X_{M}$ (or $X_{P}$ ) denotes the fundamental vector field of the action of $G$ on $M$ (or $P$ ), corresponding to $X \in \mathfrak{g}$, and $\pi^{*} f$ is the pullback of $f$ by $\pi$ (similarly, $J^{*} X$ is the pullback of $X$ by $J$, where $X$ is treated as a linear function on $\left.\mathfrak{g}^{*}\right)$. Of course for $P=T^{*} M, \pi$ is the cotangent bundle projection.

Remark 1.1 We recall that $\mathfrak{g}^{*}$ is naturally a Poisson manifold. The Poisson structure on $M$ is zero. A Poisson map is said to be complete [3], if it sends (by pullback) functions having complete Hamiltonian vector fields on functions with the same property (such functions are called complete). 


\section{Covariant representations, extended phase spaces}

It is convenient to introduce the following terminology.

Definition 2.1 A representation of a Poisson manifold $N$ in a symplectic manifold $P$ is a complete Poisson map $\Psi$ from $P$ to $N$.

Definition 2.2 Suppose we are given an action of a Lie group $G$ on a manifold $M$. A covariant representation of $\left(M, \mathfrak{g}^{*}\right)$ in a symplectic manifold $P$ is a pair $(\pi, J)$, where $\pi$ is a representation of $M$ in $P, J$ is a representation of $\mathfrak{g}^{*}$ in $P$ and the condition of covariance (4) is satisfied.

An example of a covariant representation was presented in the previous section. In fact, it has one more important property: it cannot be 'decomposed' onto smaller 'subrepresentations', because

$$
X_{P}, \mathrm{X}_{\pi^{*} f}(\text { with } X \in \mathfrak{g}, f \in M) \operatorname{span} T P \text {. }
$$

Here $\mathrm{X}_{h}$ denotes the Hamiltonian vector field of the function $h$. Note that $X_{P}=\mathrm{X}_{J^{*} X}$ for $X \in \mathfrak{g} \simeq\left(\mathfrak{g}^{*}\right)^{*}$ and one can replace $X_{P}$ in (5) by $\mathbf{X}_{J^{*} \phi}$ for $\phi \in C^{\infty}\left(\mathfrak{g}^{*}\right)$.

We say that a covariant representation $(\pi, J)$ of $\left(M, \mathfrak{g}^{*}\right)$ in $P$ is irreducible if condition 5 is satisfied. Similarly, a representation $\Psi$ of a Poisson manifold $N$ in a symplectic manifold $P$ is said to be irreducible, if $\mathrm{X}_{\Psi^{*} h}$ span $T P$ for $h \in C^{\infty}(N)$.

We can now introduce our fundamental definition.

Definition 2.3 By an extended phase space of a relativistic particle we mean an irreducible covariant representation of $\left(M, \mathfrak{g}^{*}\right)$, where $M$ is the Minkowski space and $G$ is the Poincaré group.

Remark 2.4 The above definition is applicable to other situations, like the de Sitter spacetime or the case of Poisson Minkowski space [1]. In the latter case one should replace $\mathfrak{g}^{*}$ by $G^{*}$ — the Poisson dual of the Poisson Poincaré group [5, 6, 7, 8], and also $J^{*} X$ in (4) - by the right-invariant 1 -form on $G^{*}$ corresponding to $X$. In all these cases one has the basic example provided by the cotangent bundle of $M$ (symplectic groupoid [9, 10, 11, 3] of $M$ in the case of general Poisson $M$, see [5, 6, 8, 12]).

In order to find all covariant representations of $\left(M, \mathfrak{g}^{*}\right)$ for a given action of $G$ on $M$, we notice that they are in 1-1 correspondence with representations of a certain Poisson manifold (similar fact is known in the theory of crossed products).

Proposition 2.5 There is a 1-1 correspondence between covariant representations $(\pi, J)$ of $\left(M, \mathfrak{g}^{*}\right)$ and representations $\Psi$ of the semi-direct Poisson product $M \rtimes \mathfrak{g}^{*}$, given by

$$
\Psi=\pi \times J
$$

$\Psi$ is irreducible if and only if $(\pi, J)$ is irreducible. 
To convince that this proposition is reasonable, recall [13] that $M \rtimes \mathfrak{g}^{*}$ is the cartesian product of $M$ and $\mathfrak{g}^{*}$ equipped with the semidirect Poisson structure defined by

$$
\left\{f_{1}, f_{2}\right\}=0, \quad\left\{X_{1}, X_{2}\right\}=\left[X_{1}, X_{2}\right], \quad\{X, f\}=X_{M} f
$$

for $f_{1}, f_{2} \in C^{\infty}(M), X_{1}, X_{2} \in \mathfrak{g}$. We choose the convention that $X \mapsto X_{M}$ is a homomorphism of Lie algebras, hence we choose the commutator in $\mathfrak{g}$ based on right-invariant vector fields.

Since irreducible representations of a Poisson manifold are just (coverings of) its symplectic leaves (this is a generalization of the familiar fact concerning the moment map of a transitive hamiltonian action), we conclude that irreducible covariant representations of $\left(M, \mathfrak{g}^{*}\right)$ are (coverings of) symplectic leaves in $M \rtimes \mathfrak{g}^{*}$.

\section{The classification of extended spaces}

In order to describe all possible extended phase spaces we have to study the structure of the Poisson manifold $M \rtimes \mathfrak{g}^{*}$ in the Minkowski-Poincaré case.

We denote by $V$ the subgroup of translations in the Poincaré group $G$. This is a normal subgroup and

$$
L:=G / V
$$

is the Lorentz group, acting naturally in $V$ - the tangent space of $M$. Any choice of $x \in M$ allows to identify $L$ with the stabilizing subgroup $G_{x}$ of $G$. We denote by $\mathfrak{l}$ and $\mathfrak{g}_{x}$ the Lie algebras corresponding to $L$ and $G_{x}$.

Proposition 3.1 The natural map

$$
M \rtimes \mathfrak{g}^{*} \ni(x, \alpha) \mapsto((x, p), S) \in T^{*} M \times \mathfrak{l}^{*},
$$

where $p$ is the restriction of $\alpha$ to $V$ and $S$ is the restriction of $\alpha$ to $\mathfrak{g}_{x} \simeq \mathfrak{l}$, is a Poisson isomorphism $\left(T^{*} M \times \mathfrak{l}^{*}\right.$ considered with its direct product Poisson structure).

Proof: Choose a basis $e_{k}$ in $V$ and set

$$
M_{k l}:=e_{k} \otimes g\left(e_{l}\right)-e_{l} \otimes g\left(e_{k}\right) \in \mathfrak{l} \subset \text { End } V .
$$

The 'right' commutators in $\mathfrak{g} \simeq V \rtimes \mathfrak{l}$ (we fix an identification $M \simeq V$ ) are given by

$$
\left[M_{j k}, M_{l n}\right]=M_{j l} g_{k n}+M_{k n} g_{j l}-M_{j n} g_{k l}-M_{k l} g_{j n}, \quad\left[M_{j k}, e_{l}\right]=-e_{j} g_{k l}+e_{k} g_{j l} .
$$

The same formulas define the Poisson brackets on $\mathfrak{g}^{*}$ :

$$
\left\{M_{j k}, M_{l n}\right\}=M_{j l} g_{k n}+M_{k n} g_{j l}-M_{j n} g_{k l}-M_{k l} g_{j n}, \quad\left\{M_{j k}, p_{l}\right\}=-p_{j} g_{k l}+p_{k} g_{j l}
$$

(the elements of $\mathfrak{g}$ are now (linear) functions on $\mathfrak{g}^{*} \simeq V^{*} \times \mathfrak{l}^{*}$ ). We have denoted $e_{k}$, viewed as functions on $V^{*}$, 'more physically' — by $p_{k}$ (the momenta).

It is easy to see that the 'cross' Poisson brackets in $M \rtimes \mathfrak{g}^{*}$ are given by

$$
\left\{M_{j k}, x^{l}\right\}=-x_{j} \delta_{k}{ }^{l}+x_{k} \delta_{j}^{l}, \quad\left\{p_{j}, x^{l}\right\}=\delta_{j}^{l},
$$


where $x^{l}$ are coordinates on $M \simeq V$ (corresponding to $e_{l}$ ) and $x_{k}=g_{k l} x^{l}$ (summation convention).

The transformation $(x,(p, M)) \mapsto((x, p), S)$ is given in terms of coordinates by

$$
S_{j k}=M_{j k}-p_{j} x_{k}+p_{k} x_{j} .
$$

Now it is easy to see that $\left\{S_{j k}, x^{l}\right\}=0,\left\{S_{j k}, p_{l}\right\}=0$ and

$$
\left\{S_{j k}, S_{l n}\right\}=S_{j l} g_{k n}+S_{k n} g_{j l}-S_{j n} g_{k l}-S_{k l} g_{j n}
$$

The latter equality follows easily from

$$
\left\{w_{j k}, w_{l n}\right\}=w_{j l} g_{k n}+w_{k n} g_{j l}-w_{j n} g_{k l}-w_{k l} g_{j n},
$$

where $w_{j k}:=p_{j} x_{k}-p_{k} x_{j}$ (a consequence of the fact that $w_{j k}$ describes the canonical momentum mapping for the action of $L$ on $\left.T^{*} M=T^{*} V\right)$, and

$$
\left\{M_{j k}, w_{l n}\right\}=w_{j l} g_{k n}+w_{k n} g_{j l}-w_{j n} g_{k l}-w_{k l} g_{j n} .
$$

Corollary. Extended phase spaces are of the form

$$
P=T^{*} M \times \mathcal{O},
$$

where $\mathcal{O}$ is a coadjoint orbit in $\mathfrak{l}^{*}$. They are in one-to-one correspondence with these coadjoint orbits. The trivial coadjoint orbit yields simply $T^{*} M$ - the extended phase space of a spinless particle, described in Sect. 1.

Now we recall some basic facts concerning the Lorentz Lie algebra $\mathfrak{l}$. By definition, $\mathfrak{l} \subset$ End $V$ is the orthogonal Lie algebra of the Lorentz metric $g$ of signature $(1,3)$ in $V$. The map

$$
\text { id } \otimes g: V \otimes V \rightarrow V \times V^{*} \equiv \text { End } V
$$

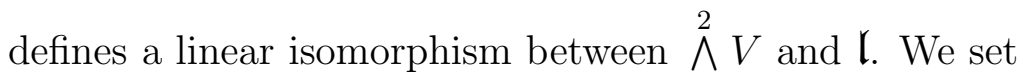

$$
x \wedge_{g} y:=x \otimes g(y)-y \otimes g(x)=(\mathrm{id} \otimes g)(x \wedge y)
$$

for $x, y \in V$.

We shall identify $\mathfrak{l}$ with its dual — our 'spin variable' $S$ will then take values in $\mathfrak{l}$ using the invariant form

$$
<S, S>:=\frac{1}{2} \operatorname{tr} S^{2}
$$

Any 'timelike' vector $u \in V$ such that $g(u, u)=1$ defines the orthogonal decomposition of $\mathfrak{l}$ on 'rotations' and 'boosts':

$$
\begin{gathered}
\mathfrak{l}=\mathfrak{l}_{u}+\left(\mathfrak{l}_{u}\right)^{\perp}, \\
S=\left(S-S u \wedge_{g} u\right)+S u \wedge_{g} u \quad \text { for } S \in \mathfrak{l} .
\end{gathered}
$$

The boost part, $S u \wedge_{g} u$, is encoded in the vector $v:=S u$ belonging to $u^{\perp}$ — the orthogonal complement of $u$. The rotation part, $S-S u \wedge_{g} u$, is also encoded in a vector $\omega \in u^{\perp}$, such that

$$
\left(S-S u \wedge_{g} u\right) x=\omega \times x \quad \text { for } x \in u^{\perp},
$$


where $\times$ denotes the three-dimensional vector product in $u^{\perp}$ (suppose we fix an overall orientation). Therefore, we can represent $S \in \mathfrak{l}$ by a pair of vectors, $(\omega, v)$, where $\omega, v \in u^{\perp}$. Using the bijection between $\mathfrak{l}_{u}$ and $\left(\mathfrak{l}_{u}\right)^{\perp}$ (both are isomorphic to $u^{\perp}$ ), one can see that $\mathfrak{l} \simeq\left(\mathfrak{l}_{u}\right)^{\mathbb{C}}$. The appropriate complex structure on $\mathfrak{g}$ is given by the following 'multiplication by $i$ :

$$
J(\omega, v):=(-v, \omega) .
$$

We conclude that $\omega$ is calculated from $S$ as follows: $\omega=(J S) u$ (recall that $v=S u$ ).

In terms of components $\omega, v$, the Killing form reads

$$
<S, S>=g(\omega, \omega)-g(v, v)=-\left(\vec{\omega}^{2}-\vec{v}^{2}\right),
$$

where $\vec{\omega}^{2}:=-g(\omega, \omega)$ denotes the positive definite metric in the three-dimensional space. We have

$$
<J S, S>=-2 g(\omega, v)=2 \vec{\omega} \cdot \vec{v},
$$

and the complex invariant form on $\mathfrak{l}$ which extends the previous real form on $\mathfrak{l}_{u}$ is given by

$$
<S, S>\mathbb{C}=<S, S>-i<J S, S>=-\left(\vec{\omega}^{2}-\vec{v}^{2}\right)-2 i \vec{\omega} \cdot \vec{v}=-(\vec{\omega}+i \vec{v})^{2} .
$$

Knowing that $\mathfrak{l} \simeq \operatorname{sl}(2, \mathbb{C})$, one can easily see that the value of the complex Killing fully specifies the adjoint orbit (if we consider only nontrivial orbits). Therefore, for any complex number $z=a+i b$ we have the orbit

$$
\mathcal{O}_{z}:=\left\{S \in \mathfrak{l} \backslash\{0\}:<S, S>_{\mathbb{C}}=-z^{2}\right\}=\left\{(\omega, v): \begin{array}{rl}
\vec{\omega}^{2}-\vec{v}^{2} & =a^{2}-b^{2} \\
\vec{\omega} \cdot \vec{v} & =a b
\end{array}\right\}
$$

( $z$ and $-z$ correspond to the same orbit). Each such orbit is of dimension 4. Corresponding extended phase spaces are 12-dimensional.

\section{$4 \quad$ Fixing spin and mass}

Each extended phase space $P_{z}=T^{*} M \times \mathcal{O}_{z}$ decomposes onto orbits of the Poincaré group. Generically, they are obtained by fixing values of the two invariants: spin and mass. We shall discuss only the case of the positive value of the mass square:

$$
m^{2}:=g(p, p)>0 .
$$

For simplicity, we identify the momentum $p \in V^{*}$ with the corresponding vector $g^{-1}(p) \in V$. The spin $s$ is given by the following expression

$$
s^{2}=-g((J S) u,(J S) u)=\vec{\omega}^{2},
$$

where $u:=\frac{p}{m}$ is the unit vector in the direction of $p$ (recall that the Pauli-Lubanski vector is defined by $W=(J S) p)$.

Fixing $m$ and $s$, we obtain generically a 10-dimensional coisotropic submanifold ('spin and mass shell') in $P_{z}$. The characteristic foliation on this submanifold is therefore 2dimensional. In order to find the leaves of this foliation, it is sufficient to integrate the hamiltonian vector fields of $\frac{1}{2} m^{2}$ and $\frac{1}{2} s^{2}$. Since

$$
\left\{\frac{1}{2} m^{2}, x\right\}=p, \quad\left\{\frac{1}{2} m^{2}, p\right\}=0, \quad\left\{\frac{1}{2} m^{2}, S\right\}=0,
$$


the mass constraint generates the usual rectilinear motion with conserved $p$ and $S$ and four-velocity $u$. In order to calculate the Poisson brackets with $\frac{1}{2} s^{2}$, note, that

$$
s^{2}=g\left(S^{2} u, u\right)+<S, S>.
$$

A simple calculation yields then

$$
\left\{\frac{1}{2} s^{2}, p\right\}=0, \quad\left\{\frac{1}{2} s^{2}, x\right\}=\frac{1}{m}\left(S^{2} u \wedge_{g} u\right) u, \quad\left\{\frac{1}{2} s^{2}, S\right\}=-S^{2} u \wedge_{g} u \in\left(\mathfrak{l}_{u}\right)^{\perp}
$$

(in order to compute the last Poisson brackets, note, that the matrix elements $S^{j}{ }_{k}$ of $S$, which are functions on $\mathfrak{l}$, correspond via the chosen invariant form to $-g^{j l} e_{l} \wedge_{g} e_{k}$ hence their Poisson brackets are minus the standard ones (7)). It follows that $p$ and $\omega$ (the rotational part of $S)$ are conserved. Since $S^{2} u=S v=\vec{\omega} \times \vec{v}+\lambda u$, we have $S^{2} u \wedge_{g} u=(\vec{\omega} \times \vec{v}) \wedge_{g} u$, hence the flow of $\frac{1}{2} s^{2}$ changes $v$ (the boost part of $S$ ) according to

$$
\left\{\frac{1}{2} s^{2}, \vec{v}\right\}=\vec{\omega} \times \vec{v}
$$

It means that $\vec{v}$ simply rotates around the $\vec{\omega}$ axis. Since

$$
\left\{\frac{1}{2} s^{2}, x+\frac{1}{m} S u\right\}=0,
$$

vector

$$
\bar{x}:=x+\frac{1}{m} S u,
$$

is conserved, and $x$ moves on a circle around the axis passing through $\bar{x}$ in the direction of $\vec{\omega}$ :

$$
x=\bar{x}-\frac{1}{m} S u=\bar{x}-\frac{1}{m} v .
$$

We conclude that the characteristics have the form of a 2-dimensional cylinder. Their projections on the Minkowski space-time are then 'world tubes' rather than 'world lines'. Each of these world tubes corresponds to a point of the coadjoint orbit in $\mathfrak{g}^{*}$ (with the fixed value of $m$ and $s$ ) and should represent the 'history' of the elementary system. In this sense we have obtained 'two-dimensional trajectories'. In a co-moving frame, an observer should see a circle of the radius $r$ with

$$
r^{2}=\frac{\left(\vec{v}^{\perp}\right)^{2}}{m^{2}}=\frac{1}{m^{2}}\left(\vec{v}^{2}-\frac{(\vec{\omega} \cdot \vec{v})^{2}}{\vec{\omega}^{2}}\right)=\frac{1}{m^{2} s^{2}}\left(s^{2}\left(s^{2}-a^{2}+b^{2}\right)-a^{2} b^{2}\right)
$$

$\left(\vec{v}^{\perp}\right.$ denotes the component of $\vec{v}$ perpendicular to $\left.\vec{\omega}\right)$, i.e.

$$
r^{2}=\frac{1}{m^{2} s^{2}}\left(s^{2}-a^{2}\right)\left(s^{2}+b^{2}\right) .
$$

For a fixed radius $r$ and orbit $\mathcal{O}_{z}, z=a+b i$, the above equation imposes a relation between spin and mass, asymptotically linear (Regge trajectory?). 


\section{Quantization}

The scheme we have presented is sufficiently universal in order to describe immediately the quantum case. Let us consider for example the case $z=0$. One can show that

$$
\mathcal{O}_{0} \simeq T^{*} \mathcal{S}^{2} \backslash \mathcal{S}^{2}
$$

- the cotangent bundle to the 2-sphere without the image of the zero section, where the cotangent bundle polarization corresponds to an invariant polarization on $\mathcal{O}_{0}$. The sphere $\mathcal{S}^{2}$ here is in fact the celestial sphere (projective forward light cone in $V$ ). Neglecting the measure zero set we have

$$
P_{0}=T^{*} M \times \mathcal{O}_{0} \simeq T^{*}\left(M \times \mathcal{S}^{2}\right),
$$

whose quantum counterpart is

$$
L^{2}(M) \otimes L^{2}\left(\mathcal{S}^{2}\right)=L^{2}\left(M \times \mathcal{S}^{2}\right) .
$$

Now the wave equations corresponding to the 'mass and spin shell' are simply obtained by replacing the classical quantities by the quantum ones:

$$
\left\{\begin{aligned}
\square_{x} \psi(x, \theta) & =m^{2} \psi(x, \theta) \\
\hat{W}^{2} \psi(x, \theta) & =m^{2} s(s+1) \psi(x, \theta)
\end{aligned}\right.
$$

Here $\square_{x}$ is the d'Alembert operator with respect to the $x$ variable, $\theta$ denotes the variable on $\mathcal{S}^{2}$ and $\hat{W}^{2}$ arises from

$$
\begin{gathered}
W^{2}=-g(W, W)=-g((J S) p,(J S) p)=g\left((J S)^{2} p, p\right)=g\left(S^{2} p, p\right)+<S, S>g(p, p) \\
=-g_{j k} S^{j}{ }_{l} p^{l} S^{k}{ }_{n} p^{n}+<S, S>m^{2}
\end{gathered}
$$

by replacing $p_{k}$ by $-i \frac{\partial}{\partial x^{k}}$ and $S^{j}$ by the generators of the representation of the Lorentz group $L$ in $L^{2}\left(\mathcal{S}^{2}\right)$.

The simplest orbit $\mathcal{O}_{0}$ corresponds to a unitary representation of the Lorentz group which contains only integral spins (the minimal spin is zero). In order to be able to pick up also half-integral spins, one has to consider other orbits/representations. Orbits $\mathcal{O}_{z}$ with $z \neq 0$, are known to be lagrangian bundles over $\mathcal{S}^{2}$ (affine bundles modelled on $T^{*} \mathcal{S}^{2}$ ). Using geometric quantization, one can construct (for quantizable orbits, i.e. for $a$ being half-integer) the corresponding unitary representations of the Lorentz group (or, rather its universal cover, $S L(2, \mathbb{C})$ ) and then wave functions on $\mathcal{S}^{2}$ have to be replaced by sections of a suitable complex line bundle over $\mathcal{S}^{2}$. This way one obtains the principal series of representations of $S L(2, \mathbb{C})$ numbered by two parameters: one discrete and one continuous. The orbit $\mathcal{O}_{z}, z=a+b i$, corresponds to the unitary representation of $S L(2, \mathbb{C})$ induced from the representation of the parabolic subgroup:

$$
\left(\begin{array}{cc}
\lambda & 0 \\
\mu & \lambda^{-1}
\end{array}\right) \mapsto|\lambda|^{2 i b}\left(\frac{\lambda}{|\lambda|}\right)^{-2 a}=\lambda^{-2 a}(\lambda \bar{\lambda})^{a+b i}
$$

( $a$ is half-integer). 
Remark. In order to obtain the quantum case, it is not necessary to consider first the classical case and then to worry about correct quantization. We can just consider any irreducible unitary representation of $L$ in a Hilbert space $H$, and pick up (by any means) a (generalized) irreducible subrepresentation of $G$ in $L^{2}(M) \otimes H$, which, essentially, amounts again to equations (8).

Problem: What is the direct relation between solutions of (8) and solutions of wave equations in some standard formulation?

\section{Fixing spin only}

It is natural to look for a possibility to fix spin first in order to obtain a 10-dimensional reduced symplectic manifold. In this manifold we could then consider pure mass shell (being more close to the concept of a wave equation in the traditional sense).

We thus consider the submanifold

$$
\mathcal{C}_{z, s}=\{\operatorname{spin}=s\} \subset\left(P_{z}\right)_{+}=\left(T^{*} M\right)_{+} \times \mathcal{O}_{z} \subset P_{z}
$$

of a fixed spin. Here $\left(T^{*} M\right)_{+}=M \times V_{+}^{*}$ is the subset of $T^{*} M$ corresponding to time-like momenta.

We recall that the characteristics on $\mathcal{C}_{z, s}$ are topological circles whose projection on $M$ are circles (in the co-moving frame) of the radius $r$ given by

$$
r^{2}=\frac{1}{m^{2} s^{2}}\left(s^{2}-a^{2}\right)\left(s^{2}+b^{2}\right) .
$$

It follows that the spin function is bounded from below on $\left(P_{z}\right)_{+}$:

$$
s \geq|a| .
$$

We have then two cases.

1. $s>|a|$. In this case $r>0$, hence $\mathbf{X}_{s^{2}} \neq 0$ (characteristics really exist), $d\left(s^{2}\right) \neq 0$ and $\operatorname{dim} \mathcal{C}_{z, s}=11\left(\mathcal{C}_{z, s}\right.$ is coisotropic). The projections of characteristics on $M$ are 'circles' (not points), therefore the variables $x^{k}$ do not pass to the quotient

$$
P_{z, s}:=\mathcal{C}_{z, s} /\{\text { circles }\}
$$

Still, the 'renormalized' position $\bar{x}=x-\frac{1}{m} S u$ is of course well defined on $P_{z, s}$. Since we have coisotropic constraints, the Poisson bracket of $\bar{x}^{j}$ and $\bar{x}^{k}$ in $P_{z, s}$ is equal to their Poisson bracket in $P_{z}$. The calculation gives

$$
\left\{\bar{x}^{j}, \bar{x}^{k}\right\}=\frac{1}{m^{2}}\left(S^{j k}-(S u)^{j} u^{k}+(S u)^{k} u^{j}\right)=\frac{1}{m^{2}} R^{j k}, \quad\left(m^{2} \equiv p^{2}\right)
$$

where $R:=S-S u \wedge_{g} u$ is the rotation part of $S$ (with respect to $u$ ). The full description of $P_{z, s}$ can be given in terms of $\bar{x}^{j}, p_{k}, R \in \mathfrak{l}$ ( such that $R u=0,<R, R>=-s^{2}$ ) and Poisson brackets

$$
\left\{p_{k}, \bar{x}^{j}\right\}=\delta_{k}^{j}, \quad\left\{p_{k}, p_{j}\right\}=0, \quad\left\{\bar{x}^{j}, \bar{x}^{k}\right\}=\frac{1}{m^{2}} R^{j k}, \quad\left\{p_{k}, R_{j n}\right\}=0,
$$




$$
\left\{R_{j k}, \bar{x}^{l}\right\}=\frac{1}{m}\left(R_{k l} u_{j}-R_{j l} u_{k}\right), \quad\left\{R_{j k}, R_{l n}\right\}=-\left(R_{j l} \tilde{g}_{k n}+R_{k n} \tilde{g}_{j l}-R_{j n} \tilde{g}_{k l}-R_{k l} \tilde{g}_{j n}\right),
$$

where $\tilde{g}_{j k}=g_{j k}-u_{j} u_{k}$ is the three-dimensional metric.

2. $s=|a|$. In this case $\vec{\omega}^{2}=a^{2}$, hence $\vec{v}^{2}=b^{2}$. Since $\vec{\omega} \cdot \vec{v}=a b, \vec{v} \| \vec{\omega}$ and it is easy to see that $\operatorname{dim} \mathcal{C}_{z, s}=10$. Calculating the symplectic form in $P_{z, s}$ on vectors tangent to $\mathcal{C}_{z, s}$ we obtain the following results

(a) for $a \neq 0, \mathcal{C}_{z, s}$ is a symplectic (sub)manifold (second class constraints!) and

$$
\left\{x^{j}, x^{k}\right\}=\frac{1}{m^{2}}\left(1+\frac{b^{2}}{a^{2}}\right) R^{j k} .
$$

In particular, when $b=0,\left\{x^{j}, x^{k}\right\}=\frac{1}{m^{2}} R^{j k}=\frac{1}{m^{2}} S^{j k}(R=S$ in this case).

(b) for $a=0, \mathcal{C}_{z, s}$ is coisotropic and $P_{z, s} \simeq T^{*} M$ (the spinless case).

\section{Conclusions}

We have constructed extended phase spaces as symplectic manifolds endowed with a Hamiltonian action of the Poincaré group and carrying a localization structure. Trajectories of an elementary system are characteristics on the 'spin and mass' shell in the extended phase space. They are typically 2-dimensional, due to the fact that we impose two constraints. The value of spin is related to the radius $r$ of the world tube. For big values of $s$, or for $P_{0}$, this relation looks as follows

$$
s=m r .
$$

This reminds the orbital angular momentum of a particle with (effective, not rest) mass $m$ moving on a circle of radius $r$ with the velocity of light $(c=1)$.

An attempt to introduce an 'intermediary extended phase space' (by a reduction with respect to a fixed spin) which would be still fibered over space-time (to this end we have to modify the original fibration), leads to 'non-commutative' space-time. The noncommutativity holds between the coordinates in the two-dimensional subspace orthogonal to the four-velocity and the actual direction of spin (the subspace of rotation). The proper angular momentum plays therefore the role of the 'source of non-commutativity'.

In the case of the extreme value of spin on $P_{z}$, the original fibration over space-time does not have to be modified. However, since the reduction is not coisotropic in this case, the original positions no longer commute (as functions on the constraint manifold). The commutation rules have here the form similar to the previous ones, with spin being the source of the non-commutativity. Since the reduction is not coisotropic, we do not have, unlike before, the 'mechanical' explanation of the non-commutativity in terms of replacing the commuting control parameters (canonical positions) by new control parameters (kinetic positions), no longer commuting, chosen for the reason of good transformation properties (in this connection, see also [6]). 


\section{References}

[1] J.-M. Souriau, Structure des systèmes dynamiques, Dunod, Paris, 1970.

[2] S. Benenti and W.M. Tulczyjew, The geometrical meaning and globalization of the Hamilton-Jacobi method, in: "Differential Geometrical Methods in Mathematical Physics", Proceedings, Aix-en-Provence and Salamanca 1979, Springer (Berlin), Lecture Notes in Mathematics 836, 1980, pp. 9-21.

[3] S. Zakrzewski, Quantum and classical pseudogroups, Commun. Math. Phys. 134 (1990), 347-395.

[4] S. Zakrzewski, Poisson homogeneous spaces, Proceedings of the Karpacz Winter School on Theoretical Physics 1994, to appear.

[5] S. Zakrzewski, Poisson space-time symmetry and corresponding elementary systems, in: "Quantum Symmetries", Proceedings of the II International Wigner Symposium, Goslar 1991, H.D. Doebner and V.K. Dobrev (Eds.), 1993, pp. 111-123.

[6] S. Zakrzewski, Poisson Poincaré particle and canonical variables, in: "Generalized Symmetries in Physics", Proceedings of the International Symposium on Mathematical Physics, Clausthal, July 27-29, 1993, H.-D. Doebner, V.K. Dobrev and A.G. Ushveridze (Eds.), 1994, pp. 165-171.

[7] S. Zakrzewski, Poisson Poincaré groups, Proceedings of the Karpacz Winter School on Theoretical Physics 1994, to appear.

[8] S. Zakrzewski, On the classical $\kappa$-particle, Proceedings of the Karpacz Winter School on Theoretical Physics 1994, to appear.

[9] A. Weinstein, Symplectic groupoids and Poisson manifolds, Bull. Amer. Soc. 16 (1987), 101-104.

[10] A. Coste, P. Dazord and A. Weinstein, Groupoïdes symplectiques, Publ. du Dép. de Math. de l'Université de Lyon 1, 2/A- 1987, 1-62

[11] M. V. Karasev, Analogues of objects of Lie group theory for nonlinear Poisson brackets, Math. USSR Izvestiya 28 (1987), 497-527.

[12] S. Zakrzewski, Geometric quantization of Poisson groups - diagonal and soft deformations, Proceedings of the Taniguchi Symposium Symplectic geometry and quantization problems, Sanda (1993), Y. Maeda, H. Omori and A. Weinstein (Eds.), Contemporary Mathematics 179, 1994, 271-285.

[13] A. Weinstein, Poisson geometry of the principal series and nonlinearizable structures, J. Diff. Geom. 25 (1987), 55-73. 